\title{
Concentração eficiente e econômica de caulim para a proteção de algodoeiro contra o bicudo
}

\author{
Ana Lígia Aureliano de Lima e Silva( ${ }^{(1)}$ e Carlos Alberto Domingues da Silva(2)
}

\begin{abstract}
(1)Universidade Estadual da Paraíba, Rua Baraúnas, № 351, Bairro Universitário, CEP 58429-000, Campina Grande, PB, Brasil. E-mail: ana.ligiaaureliano@hotmail.com (2)Embrapa Algodão, Caixa Postal 174, CEP 58428-095, Campina Grande, PB, Brasil. E-mail: carlos.domingues-silva@embrapa.br
\end{abstract}

\begin{abstract}
Resumo - O objetivo deste trabalho foi determinar a concentração de caulim mais eficiente e econômica para a proteção de algodoeiro contra os danos causados pelo bicudo-do-algodoeiro (Anthonomus grandis). Utilizou-se o delineamento experimental de blocos ao acaso, com cinco tratamentos de pulverizações com caulim nas concentrações: 20, 40, 60, 80 e $100 \mathrm{~g} \mathrm{~L}^{-1}$. Determinaram-se os seguintes parâmetros: percentagem de botões florais com orifícios de oviposição por fêmeas do bicudo; resíduos de caulim depositados sobre as folhas e as brácteas do algodoeiro; média da produção; e receita líquida do algodão, por hectare, a partir da pesagem da pluma de algodão com caroço. As percentagens de botões florais com orifício de oviposição variaram de 13,6 a $30,8 \%$; o resíduo médio de caulim depositado nas folhas, de 0,0010 a $0,0034 \mathrm{mg} \mathrm{mm}^{-2}$, e nas brácteas, de 0,0010 a $0,0034 \mathrm{mg} \mathrm{mm}^{-2}$; a produção variou de 348,1 a $717,8 \mathrm{~kg} \mathrm{ha}^{-1}$; e a receita líquida de algodão de $\mathrm{R} \$ 1.033,88$ a $\mathrm{R} \$ 2.098,86$ por hectare. As concentrações de caulim mais eficientes para a proteção de algodoeiro contra o bicudo são as de 60,80 e $100 \mathrm{~g} \mathrm{~L}^{-1}$; no entanto, a de $60 \mathrm{~g} \mathrm{~L}^{-1}$ foi a mais econômica.

Termos para indexação: Anthonomus grandis, Gossypium hirsutum, algodão, manejo integrado de pragas, pulverização.

\section{Efficient and economical kaolin concentration for cotton protection against boll weevil}

\begin{abstract}
The objective of this work was to determine the most effective and economical kaolin concentration to protect cotton against the injuries caused by the boll weevil (Anthonomus grandis). A randomized complete block design was used with five treatments of kaolin spraying at the following concentrations: 20, 40, 60, 80, and $100 \mathrm{~g} \mathrm{~L}^{-1}$. The following parameters were determined: percentage of punctured squares with oviposition by boll weevil females; the amount of kaolin residues on leaves and bracts of cotton square; average yield; and the cotton net income per hectare, measured from the weighing of cotton lint with seed. The percentages of oviposition-punctured squares ranged from 13.6 to $30.8 \%$; kaolin residue, deposited on cotton leaves, ranged from 0.0010 to $0.0034 \mathrm{mg} \mathrm{mm}^{-2}$, and on bracts, from 0.0010 to $0.0034 \mathrm{mg} \mathrm{mm}^{-2}$; production ranged from 348.1 to $717.8 \mathrm{~kg} \mathrm{ha}^{-1}$; and the net revenues from cotton ranged from $\mathrm{R} \$ 1,033.88$ to $\mathrm{R} \$ 2,098.86$ per hectare. The most efficient kaolin concentrations for cotton protection against damage caused by boll weevil are those of 60 , 80 , and $100 \mathrm{~g} \mathrm{~L}^{-1}$; however, the concentration of $60 \mathrm{~g} \mathrm{~L}^{-1}$ is the most economic one.
\end{abstract}

Index terms: Anthonomus grandis, Gossypium hirsutum, cotton, integrated pest management, spraying.

\section{Introdução}

O bicudo, Anthonomus grandis grandis Boheman (Coleoptera: Curculionidae), é um inseto originário da Mesoamérica que se encontra distribuído em cultivos de algodoeiro, Gossypium hirsutum L., desde o Sul dos Estados Unidos até o Norte da Argentina (Showler, 2007). Desde sua introdução em meados da década de 1980, esse inseto tem sido responsável por ocasionar enormes prejuízos econômicos aos cotonicultores brasileiros (Paula et al., 2013).
Os bicudos são atraídos para as lavouras de algodão por pistas visuais e voláteis, emitidos pelas plantas logo após a formação dos botões florais (Showler, 2005) e, em particular, pelo feromônio de agregação de outros bicudos presentes no campo (Parajulee \& Slosser, 2001; Showler \& Robinson, 2005). Após se alimentar dos botões florais, flores e maçãs do algodoeiro, a fêmea oviposita no interior dessas estruturas vegetais, que suas larvas destroem internamente, provocando sua queda e acarretando redução da produtividade em até 54 e 87\% (Silva \& Ramalho, 2013). 
O bicudo é controlado, principalmente, por meio de aplicações de inseticidas químicos e, apesar da elevada eficiência desses produtos, seu uso intensivo pode ocasionar diversos problemas, como poluição ambiental, toxicidade a organismos não alvos $\mathrm{e}$ o desenvolvimento da resistência pelas pragas (Ogendo et al., 2008). Por isso, produzir algodão de forma agroecológica é um dos grandes desafios da agricultura sustentável. Assim, todas as estratégias que possibilitem melhoria dos retornos obtidos com as culturas presentes no cultivo agroecológico devem ser compatíveis com a redução da competição imposta por artrópodes fitófagos (Beltrão et al., 2009).

A tecnologia do filme de partículas de caulim temse mostrado eficiente na redução de populações de várias pragas-chave do algodoeiro, inclusive bicudos, pulgões, mosca-branca, lagarta-rosada e o complexo de lagartas-das-maçãs e do gênero Spodoptera (Showler, 2002, 2003; Sisterson et al., 2003; Alavo, 2006; Alavo et al., 2010, 2011; Silva \& Ramalho, 2013; Neves et al., 2014).

O caulim impede o contato visual e táctil do bicudo com a planta de algodão e, consequentemente, acaba por retardar a colonização da lavoura e prejudicar o comportamento de alimentação e oviposição desse coleóptero nas estruturas reprodutivas do algodoeiro (Showler, 2002; Silva \& Ramalho, 2013). Esse produto, aplicado à concentração de $60 \mathrm{~g} \mathrm{~L}^{-1}$, reduz consideravelmente os prejuízos causados pelo bicudo às lavouras de algodão do Semiárido brasileiro (Silva \& Ramalho, 2013; Neves et al., 2014). Apesar disso, faltam informações sobre o grau de proteção de algodoeiros ao ataque dessa praga, em resposta a outras concentrações desse produto.

$\mathrm{O}$ objetivo deste trabalho foi determinar a concentração de caulim mais eficiente e econômica, para a proteção de algodoeiros contra os prejuízos causados pelo bicudo-do-algodoeiro.

\section{Material e Métodos}

O estudo foi realizado no campo da Estação Experimental da Embrapa Algodão, em Campina Grande, PB, Brasil, a $7^{\circ} 13^{\prime} 50^{\prime \prime S}$ e $35^{\circ} 52^{\prime} 52^{\prime \prime} \mathrm{W}$.

Utilizou-se um delineamento experimental de blocos ao acaso, com cinco tratamentos e quatro repetições. As parcelas foram constituídas por cinco linhas de algodão, com $5 \mathrm{~m}$ de comprimento.
A cultivar de algodão BRS 286 foi cultivada no campo, em área de $3.290 \mathrm{~m}^{2}(47 \times 70 \mathrm{~m})$, no espaçamento de $0,90 \times 0,20 \mathrm{~m}$, tendo-se deixado uma planta por cova após o desbaste. Aos 30 dias após a germinação, quando surgiram os primeiros botões florais, iniciaramse as pulverizações com caulim, sempre que o número de plantas danificadas pelo bicudo apresentava $5 \%$ de botões florais com orifício de oviposição, conforme recomendações de Silva \& Ramalho (2013). Os tratamentos consistiram de pulverizações sobre as plantas, com suspensão de caulim à concentração de água de: 20, 40, 60, 80 e $100 \mathrm{~g} \mathrm{~L}^{-1}$.

As pulverizações contra o bicudo foram realizadas com um pulverizador costal manual, com capacidade para $20 \mathrm{~L}$ de calda e bico $\mathrm{D}_{2}$ do tipo cone. O bico do pulverizador foi posicionado lateralmente à fileira, a cerca de $20 \mathrm{~cm}$ das plantas de algodão. A vazão foi ajustada conforme o estágio de crescimento da cultura e variou de 150 a $300 \mathrm{~L}_{\text {de água ha }}{ }^{-1}$ (Silva \& Ramalho, 2013).

As avaliações foram feitas a cada sete dias, desde o surgimento dos primeiros botões florais até o aparecimento dos capulhos, tendo-se observado, aleatoriamente, 10 plantas de algodoeiro por parcela. Em cada planta, examinou-se um botão floral de tamanho médio ( $>3 \mathrm{~mm}$ e $<6 \mathrm{~mm}$ de diâmetro), tomado aleatoriamente da metade superior da planta, tendo-se registrado a presença ou não de orifício de oviposição por fêmeas do bicudo.

Coletaram-se, também, semanalmente, neste mesmo período de avaliação, uma folha e um botão floral da região apical por planta, de cinco plantas por parcela, para estimar a quantidade de caulim depositada nessas estruturas vegetais, que foram coletadas e colocadas em sacos de papel, devidamente etiquetados, e acondicionadas em caixa de isopor com gelo com capacidade de $20 \mathrm{~L}$.

No laboratório, as folhas e as brácteas dos botões florais foram lavadas com $100 \mathrm{~mL}$ de água deionizada, e a água da lavagem foi armazenada em placas de Petri, que foram pesadas e colocadas em estufa a $80^{\circ} \mathrm{C}$, por 24 horas, para evaporação e determinação da massa do resíduo seco.

A quantidade de caulim, depositada no fundo do recipiente, foi pesada para determinação da quantidade de caulim por milímetro quadrado depositado na estrutura vegetal, conforme Cantore et al. (2009). A área foi determinada por meio do medidor portátil 
AM 300 (ADC BioScientific Ltd., Hoddesdon, Reino Unido), enquanto o peso das estruturas vegetais foi determinado por meio de uma balança analítica AY220 (Shimadzu Corporation, Columbia, MD, EUA) com divisão decimal de 0,0001 . A massa de caulim removida de cada folha e bráctea de algodão, coletada em placas de Petri, foi dividida pela área total da superfície da folha e da bráctea, para obtenção da massa de caulim depositada por milímetro quadrado.

As médias da percentagem de botões florais danificados pelo bicudo e da quantidade de caulim por milímetro quadrado de folhas e brácteas dos botões florais, nos diferentes tratamentos, durante o período avaliado, foram submetidas à análise de variância, a $5 \%$ de probabilidade, e à análise de regressão. Efetuouse, também, a análise de correlação de Pearson (r) entre a percentagem de botões florais danificados pelo bicudo e a quantidade de caulim por milímetro quadrado de folhas e brácteas dos botões florais, nos diferentes tratamentos, durante o período avaliado. Para as análises, utilizou-se o Saeg (Ribeiro Júnior, 2001).

As médias da produção e da receita líquida do algodão foram estimadas por hectare, a partir da pesagem da pluma de algodão com caroço, colhido manualmente dos capulhos, de todas as plantas da parcela, em uma área útil de $14,4 \mathrm{~m}^{2}$, multiplicado pelo preço do algodão em pluma e caroço, menos a quantidade de caulim consumida por hectare multiplicada pelo preço do quilo de caulim.

\section{Resultados e Discussão}

A análise de variância diferiu entre as cinco concentrações de caulim testadas, quanto à percentagem média total de botões florais com orifício de oviposição $\left(\mathrm{F}_{4,12}=11,72 ; \mathrm{p}<0,01\right)$, à produção $\left(\mathrm{F}_{4,12}=3,36 ; \mathrm{p}=0,05\right)$, ao resíduo de caulim depositado nas folhas $\left(\mathrm{F}_{4,44}=9,36\right.$; $\mathrm{p}<0,01)$ e brácteas $\left(\mathrm{F}_{4,44}=6,31 ; \mathrm{p}<0,01\right)$ de algodoeiro.

A percentagem média de botões florais de algodão, com orifício de oviposição, variou de 13,6 a 30,8\% durante o período de avaliação. Os percentuais de botões florais com orifício de oviposição por fêmeas do bicudo apresentaram tendência a decréscimo em relação ao aumento das concentrações de caulim: quanto maior a concentração de caulim, menor a percentagem de botões florais com orifício de oviposição (Figura 1).
Tais resultados discordam dos obtidos para Zabrotes subfasciatus (Boheman, 1833) (Coleoptera: Crysomelidae: Bruchidae) em sementes de feijão, Phaseolus vulgaris L. (Fabales: Fabaceae), tratadas com caulim à concentração de $2,4 \mathrm{e} 8 \mathrm{~g} \mathrm{~kg}^{-1}$ de semente (Mikami et al., 2010), cujas maiores doses de caulim não aumentaram significativamente a mortalidade do inseto, e dados para oviposição de Malacossoma disstria Hübner (Lepidoptera: Lasiocampidae), em folhas de Quercus rubrus L. (Fagales: Fagaceae), tratadas com caulim a 60 e $120 \mathrm{~g} \mathrm{~L}^{-1}$ (Cadogan \& Scharbach, 2005a).

No entanto, concordam com aqueles observados quanto à oviposição de Lymantria dispar (L.) (Lepidoptera: Lymantriidae) em folhas de carvalho-vermelho, $Q$. rubrus, tratadas com caulim a 60 e $120 \mathrm{~g} \mathrm{~L}^{-1}$ (Cadogan \& Scharbach, 2005a), e quanto à oviposição de Choristoneura fumiferana (Lepidoptera: Tortricidae) em brotos tríades de
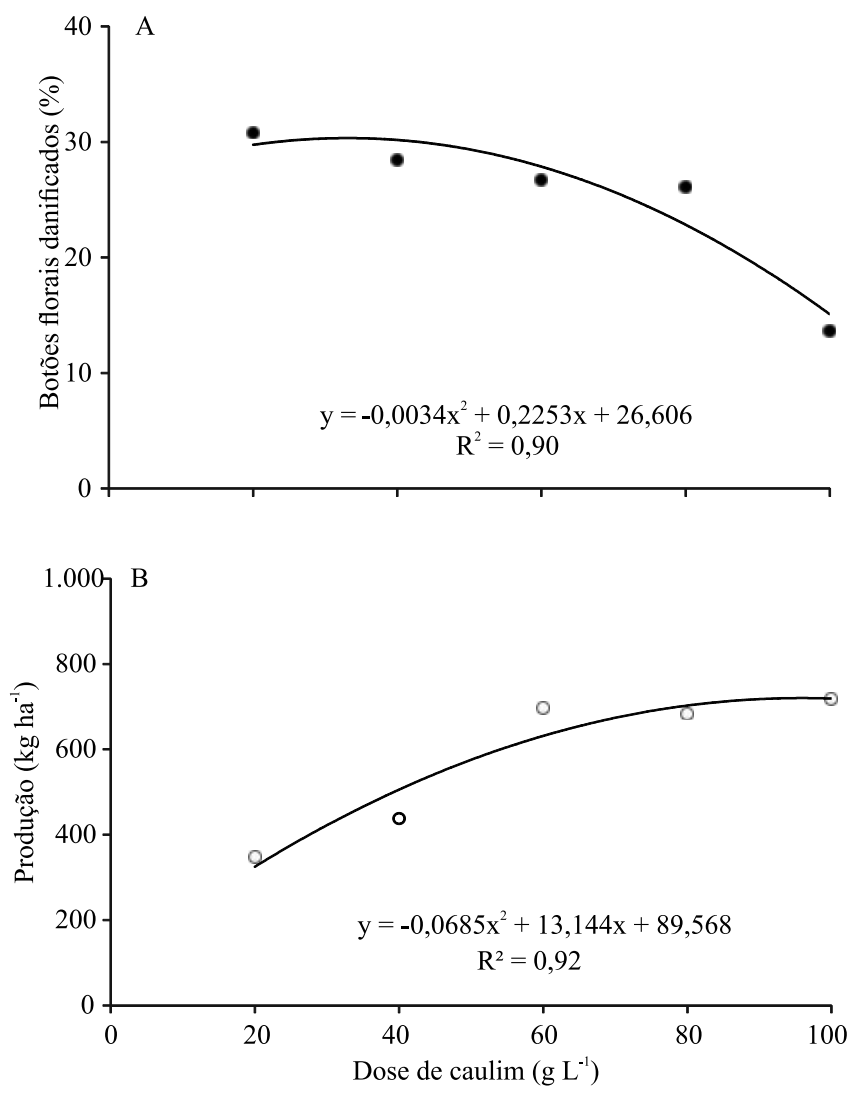

Figura 1. Média estimada dos botões florais danificados pelo bicudo (A) e a média estimada da produção de algodão em caroço (B), em função da concentração de caulim durante todo período de avaliação. 
Picea glauca (Moench) Voss (Pinales: Pinaceae), tratados com caulim a 15,30 e $60 \mathrm{~g} \mathrm{~L}^{-1}$ (Cadogan \& Scharbach, 2005b), o que indica que as variações de comportamento de postura dos insetos-praga a diferentes concentrações do caulim são específicas à espécie (Glenn \& Puterka, 2005).

A percentagem de botões florais danificados pelo bicudo apresentou correlação negativa significativa com a quantidade de caulim por milímetro quadrado de folhas $(\mathrm{r}=-5,01, \mathrm{p}<0,01)$, ou seja, o resíduo médio depositado sobre as folhas do algodoeiro, nas cinco concentrações de caulim testadas, apresentou tendência de crescimento das menores concentrações para as maiores e variou de 0,0010 a $0,0034 \mathrm{mg} \mathrm{mm}^{-2}$ (Figura 2), durante o período de avaliação.

No entanto, a percentagem de botões florais danificados pelo bicudo não apresentou correlação significativa com a quantidade de caulim por milímetro quadrado nas brácteas $(\mathrm{r}=-0,92, \mathrm{p}=0,21)$, ou seja, o efeito repelente do caulim ao bicudo não está
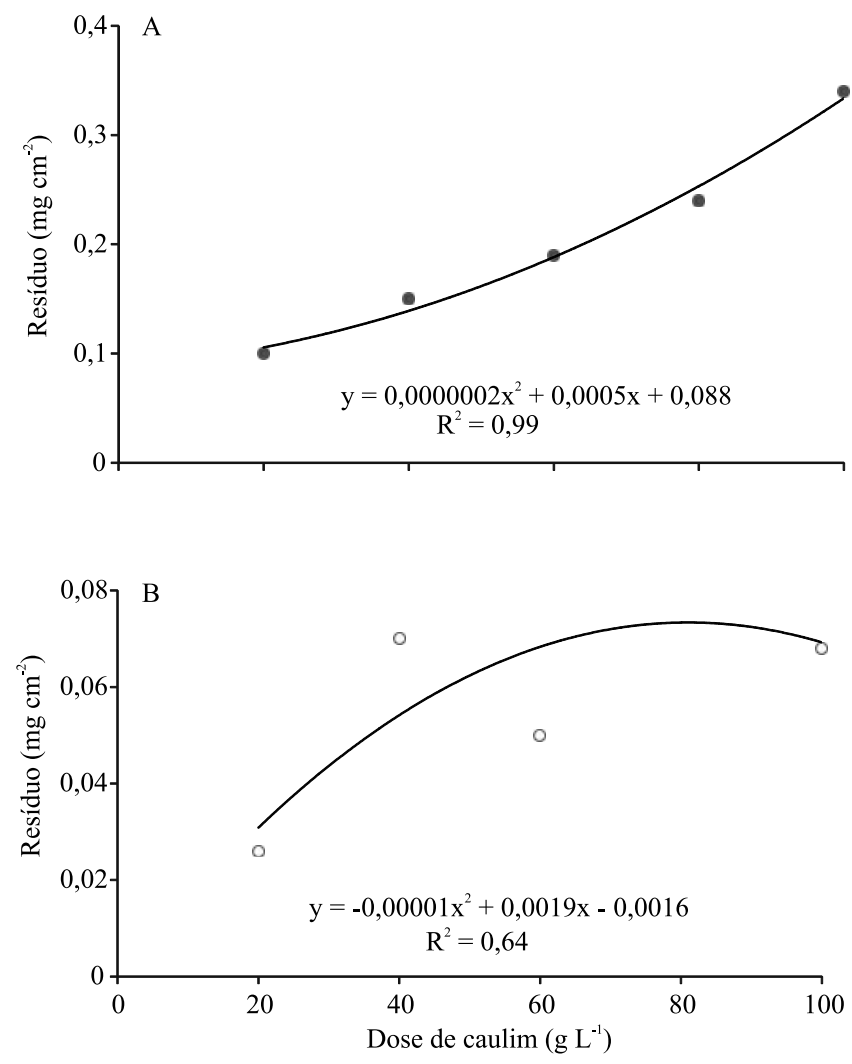

Figura 2. Média estimada de partículas de caulim sobre folhas (A) e brácteas (B) de algodão, em função da concentração durante todo o período de avaliação. relacionado ao tingimento das brácteas pela cor branca desse produto. Isto pode explicar porque o resíduo médio depositado sobre as brácteas tendeu a crescer, entre as concentrações de 20 a $80 \mathrm{~g} \mathrm{~L}^{-1}$ de caulim e, a partir daí, começou a decrescer e apresentou, à concentração de caulim de $100 \mathrm{~g} \mathrm{~L}^{-1}$, resíduo semelhante ao observado para a concentração de $40 \mathrm{~g} \mathrm{~L}^{-1}$ (Figura 2).

$\mathrm{O}$ resíduo médio de caulim nas brácteas variou de 0,0003 a $0,0008 \mathrm{mg} \mathrm{mm}^{-2}$, durante o período de avaliação. Esses resultados podem ser atribuídos às diferenças morfológicas entre as folhas e as brácteas dos botões florais do algodoeiro e, também, à quantidade e posição relativa dessas estruturas vegetais no dossel do algodoeiro. A morfologia foliar pode desempenhar papel importante na deposição da pulverização, por meio da impregnação e retenção de resíduos inseticidas em estruturas como pelos, arestas, veias, cera cuticular e outras saliências (DeRuiter et al., 1990; Himel et al., 1990). As brácteas, no entanto, representam alvos mais difíceis de serem atingidos nas pulverizações do que as folhas, pois, por apresentar menor tamanho e estar em menor número, estão protegidas da calda inseticida, pelo efeito de sobreposição das folhas no caminho da gota (Balan et al., 2005; Román, 2009).

Em termos percentuais, os resíduos médios totais de caulim recuperados das folhas e brácteas de algodoeiro após a aplicação da calda nas concentrações de 20, 40, 60,80 e $100 \mathrm{~g} \mathrm{~L}^{-1}$ foram de 0,$005 ; 0,004 ; 0,003 ; 0,003$ e $0,003 \%$ e de 0,$001 ; 0,002 ; 0,001 ; 0,001$ e $0,001 \%$ do peso do produto pulverizado, respectivamente.

Os valores de resíduos de caulim recuperados, no presente trabalho, foram proporcionalmente inferiores aos utilizados por Cadogan \& Scharbach (2005b), que recuperaram $0,4,1,1$ e $1,8 \%$ do peso do produto, após pulverizações com caulim Surround WP sobre brotos tríades de $P$. glauca a 15,30 e $60 \mathrm{~g} \mathrm{~L}^{-1}$, para o controle de $C$. fumiferana, o que pode ser atribuído às diferenças entre os espécimes vegetais e às metodologias de recuperação do resíduo de caulim utilizadas em cada experimento.

A curva de produção de algodão apresentou, em geral, tendência de crescimento das menores concentrações de caulim para as maiores e variou de 348,1 a $717,8 \mathrm{~kg} \mathrm{ha}^{-1}$, ou seja, quanto maior a concentração de caulim, maior a produção de algodão com caroço (Figura 1, Tabela 1). As maiores receitas líquidas para a produção de algodão foram estimadas em ordem crescente, para os tratamentos pulverizados com caulim a 80,60 e $100 \mathrm{~g} \mathrm{~L}^{-1}$ (Tabela 1), e as 
Tabela 1. Concentração, quantidade e preço do caulim para dez aplicações, produção de algodão em pluma e caroço, preço da produção e receita bruta e líquida do algodão(1).

\begin{tabular}{|c|c|c|c|c|c|c|c|c|}
\hline \multirow{2}{*}{$\begin{array}{l}\text { Concentração } \\
\left(\mathrm{g} \mathrm{L}^{-1}\right)\end{array}$} & \multicolumn{2}{|c|}{ Caulim } & \multicolumn{2}{|c|}{ Produção de algodão $\left(\mathrm{kg} \mathrm{ha}^{-1}\right)$} & \multicolumn{2}{|c|}{ Preço do algodão ${ }^{(2)}\left(\mathrm{R} \$\right.$ ha $\left.^{-1}\right)$} & \multicolumn{2}{|c|}{ Receita (R\$) } \\
\hline & $\begin{array}{c}\text { Quantidade } \\
\left(\mathrm{kg} \mathrm{ha}^{-1}\right)\end{array}$ & $\begin{array}{c}\text { Preço } \\
\text { (R\$) }\end{array}$ & Pluma & Caroço & $\begin{array}{c}\text { Pluma } \\
\text { (a) }\end{array}$ & $\begin{array}{l}\text { Caroço } \\
\text { (b) }\end{array}$ & $\begin{array}{l}\text { Bruta } \\
(\mathrm{a}+\mathrm{b})\end{array}$ & Líquida \\
\hline 20 & 32 & 11,20 & 131,37 & 216,75 & 466,35 & 578,73 & $1.045,08$ & $1.033,88$ \\
\hline 40 & 64 & 22,40 & 165,26 & 272,67 & 586,66 & 728,04 & $1.314,70$ & $1.292,30$ \\
\hline 60 & 96 & 33,60 & 262,94 & 433,84 & 933,42 & $1.158,36$ & $2.091,79$ & $2.058,19$ \\
\hline 80 & 128 & 44,80 & 258,05 & 425,78 & 916,07 & $1.136,84$ & $2.052,91$ & $2.008,11$ \\
\hline 100 & 160 & 56,00 & 270,86 & 446,93 & 961,57 & $1.193,29$ & $2.154,86$ & $2.098,86$ \\
\hline
\end{tabular}

${ }^{(1)} \mathrm{O}$ caulim e o algodão foram cotados, respectivamente, para exportação e comercialização, na região de Barreiras, BA. Fonte: Aliceweb (Caulim..., 2012) e Cepea/Esalq (Algodão..., 2014).

menores, em ordem decrescente, para os tratamentos com caulim a 40 e $20 \mathrm{~g} \mathrm{~L}^{-1}$. Essa receita líquida, que foi maior pela concentração de caulim a $60 \mathrm{~g} \mathrm{~L}^{-1}$ do que pela de $80 \mathrm{~g} \mathrm{~L}^{-1}$, mostra que nem sempre a maior concentração de caulim é necessariamente a mais eficiente do ponto de vista econômico.

Isto foi mostrado em estudo sobre o pulgão-do-trigo Schizaphis graminum (Rondani, 1852) (Hemiptera: Aphididae), submetido a diferentes concentrações e períodos de aplicação de caulim (Nateghi et al., 2013). Além disso, essa receita líquida maior, pelo uso da concentração a $60 \mathrm{~g} \mathrm{~L}^{-1}$, confirma que as pulverizações de caulim são capazes de retardar a colonização das lavouras de algodão pelo bicudo e, assim, reduzir seus prejuízos (Showler, 2002; Silva \& Ramalho, 2013).

Os resultados obtidos no presente estudo mostram que o caulim pulverizado sobre a lavoura de algodão, a 60,80 e $100 \mathrm{~g} \mathrm{~L}^{-1}$, é uma importante ferramenta para reduzir os prejuízos ocasionados pelo bicudo. No entanto, as pulverizações com caulim a 80 e $100 \mathrm{~g} \mathrm{~L}^{-1}$ consumiram maior quantidade do produto e ocasionaram, por algumas vezes, entupimento da peneira do bico do pulverizador, o que exigiu maiores cuidados com sua limpeza. Por isto, maior economia monetária e de produto é obtida nas aplicações de caulim a $60 \mathrm{~g} \mathrm{~L}^{-1}$ de concentração.

\section{Conclusão}

As concentrações de caulim mais eficientes para a proteção de algodoeiro, contra os prejuízos causados pelo bicudo, são as de 60,80 e $100 \mathrm{~g} \mathrm{~L}^{-1}$; no entanto, maior economia monetária e de produto é obtida com pulverizações sobre algodoeiro à concentração de $60 \mathrm{~g} \mathrm{~L}^{-1}$ de caulim.

\section{Agradecimentos}

Ao Conselho Nacional de Desenvolvimento Científico e Tecnológico (CNPq) e à Coordenação de Aperfeiçoamento de Pessoal de Nível Superior (Capes), pela concessão de bolsa.

\section{Referências}

ALAVO, T.B.C. Biological control agents and environmentally-friendly compounds for the integrated management of Helicoverpa armigera Hübner (Lepidoptera: Noctuidae) on cotton: perspectives for pyrethroid resistance management in West Africa. Archives of Phytopathology and Plant Protection, v.39, p.105-111, 2006. DOI: 10.1080/03235400500181576.

ALAVO, T.B.C.; ABAGLI, A.Z.; TÉGBÉSSOU, K.J.C.; DUNPHY, G.B. Kaolin potential for the integrated management of Aphis gossypii Glov. (Homoptera: Aphididae) on cotton. Archives of Phytopathology and Plant Protection, v.44, p.764-770, 2011. DOI: $10.1080 / 03235408.2010 .507959$.

ALAVO, T.B.C.; YAROU, B.B.; ATACHI, P. Field effects of kaolin particle film formulation against major cotton lepidopteran pests in North Benin, West Africa. International Journal of Pest Management, v.56, p.287-290, 2010. DOI: 10.1080/09670871003628389.

ALGODÃO: informações de mercado. In: AGROMENSAL. Piracicaba: Cepea, Esalq, 2014.

BALAN, M.G.; SAAB, O.J.G.A.; FONSECA, I.C. de B.; SILVA, C.G. da; SASAKI, E.H. Pulverização em alvos artificiais: avaliação com o uso do software conta-gotas. Ciência Rural, v.35, p.916-919, 2005. DOI: 10.1590/S0103-84782005000400026.

BELTRÃO, N.E. de M.; SILVA, C.A.D.; BASTOS, C.S.; SUINAGA, F.A.; ARRIEL, N.H.C.; RAMALHO, F.S. Algodão agroecológico: opção de agronegócio para o Semiárido do Brasil. Campina Grande: Embrapa Algodão, 2009. 62p. (Embrapa Algodão. Documentos, 222).

CADOGAN, B.L.; SCHARBACH, R.D. Effects of a kaolin-based particle film on oviposition and feeding of gypsy moth (Lep., 
Lymantriidae) and forest tent caterpillar (Lep., Lasiocampidae) in the laboratory. Journal of Applied Entomology, v.129, p.498-504, 2005a. DOI: 10.1111/j.1439-0418.2005.01000.x.

CADOGAN, B.L.; SCHARBACH, R.D. Effects of kaolin-based particle film on spruce budworm (Choristoneura fumiferana (Lepidoptera: Tortricidae)) oviposition in the laboratory. Pest Management Science, v.61, p.1215-1219, 2005b. DOI: 10.1002/ ps.1131.

CANTORE, V.; PACE, B.; ALBRIZIO, R. Kaolin-based particle film technology affects tomato physiology, yield and quality. Environmental and Experimental Botany, v.66, p.279-288, 2009. DOI: 10.1016/j.envexpbot.2009.03.008.

CAULIM. In: Informações e análises da economia mineral brasileira. 7.ed. Brasília: Instituto Brasileiro de Mineração, 2012. p.22-23.

DERUITER, H.D.; UFFING, A.J.M.; MEINEN, E.; PRINS, A. Influence of surfactants and plant species on leaf deposition of spray solutions. Weed Science, v.38, p.567-572, 1990.

GLENN, D.M.; PUTERKA, G.J. Particle films: a new technology for agriculture. Horticultural Review, v.31, p.1-44, 2005.

HIMEL, C.M.; LOATS, H.; BAILEY, G.W. Pesticide sources to the soil and principles of spray physics. In: CHENG, H.H. (Ed.). Pesticides in the soil environment: processes, impact, and modelling. Madison: Soil Science Society of America, 1990. p.7-50.

MIKAMI, A.Y.; PISSINATI, A.; FAGOTTI, D.; MENEZES JÚNIOR, A. de O.; VENTURA, M.U. Control of the Mexican bean weevil Zabrotes subfasciatus with kaolin. Ciência Rural, v.40, p.1497-1501, 2010. DOI: 10.1590/S0103-84782010005000108.

NATEGHI, M.; PAKNEJAD, F.; MOAREFI, M. Effect of concentrations and time of kaolin spraying on wheat aphid. Journal of Biological Environmental Science, v.7, p.163-168, 2013.

NEVES, R.C.S.; COLARES, F.; TORRES, J.B.; SANTOS, R.L.; BASTOS, C.S. Rational practices to manage boll weevils colonization and population growth on family farms in the Semiárido region of Brazil. Insects, v.5, p.818-831, 2014. DOI: 10.3390/insects5040818.

OGENDO, J.O.; KOSTYUKOVSKY, M.; RAVID, U.; MATASYOH, J.C.; DENG, A.L.; OMOLO, E.O.; KARIUKI, S.T.; SHAAYA, E. Bioatctivity of Ocimun gratissimum L. oil and two of its constituents against five insect pests attacking stored food products. Journal of Stored Products Research, v.44, p.328-334, 2008. DOI: 10.1016/j.jspr.2008.02.009.

PARAJULEE, M.N.; SLOSSER, J.E. Effect of ethephon on efficacy of grandlure-baited pheromone traps in surveying fall and spring populations of the boll weevil (Coleoptera: Curculionidae).
Environmental Entomology, v.30, p.64-69, 2001. DOI: 10.1603/0046-225X-30.1.64.

PAULA, D.P.; CLAUDINO, D.; TIMBÓ, R.V.; MIRANDA, J.E.; BEMQUERER, M.P.; RIBEIRO, A.C.J.; SUJII, E.R.; FONTES, E.M.G.; PIRES, C.S.S. Reproductive dormancy in boll-weevil from populations of the Midwest of Brazil. Journal of Economic Entomology, v.106, p.86-96, 2013. DOI: 10.1603/EC12092.

RIBEIRO JR., J.I. Análises estatísticas no SAEG. Viçosa: Ed. da UFV, 2001. 301p.

ROMÁN, R.A.A.; CORTEZ, J.W.; FERREIRA, M. da C.; OLIVEIRA, J.R.G. di. Cobertura da cultura da soja pela calda fungicida em função de pontas de pulverização e volumes de aplicação. Scientia Agraria, v.10, p.223-232, 2009. DOI: 10.5380/ rsa.v10i3.14529.

SHOWLER, A.T. Effects of kaolin particle film on beet armyworm, Spodoptera exigua (Hübner) (Lepidoptera: Noctuidae), oviposition, larval feeding and development on cotton, Gossypium hirsutum L. Agriculture, Ecosystems and Environment, v.95, p.265-271, 2003. DOI: 10.1016/S0167-8809(02)00101-9.

SHOWLER, A.T. Effects of kaolin-based particle film application on boll weevil (Coleoptera: Curculionidae) injury to cotton. Journal of Economic Entomology, v.95, p.754-762, 2002. DOI: 10.1603/0022-0493-95.4.754.

SHOWLER, A.T. Relationships of different cotton square sizes to boll weevil (Coleoptera: Curculionidae) feeding and oviposition in field conditions. Journal of Economic Entomology, v.98, p.1572-1579, 2005. DOI: 10.1093/jee/98.5.1572.

SHOWLER, A.T.; ABRIGO, V. Common subtropical and tropical nonpollen food sources of the boll weevil (Coleoptera: Curculionidae). Environmental Entomology, v.36, p.99-104, 2007. DOI: 10.1093/ee/36.1.99.

SHOWLER, A.T.; ROBINSON, J.R.C. Proactive spraying against boll weevils (Coleoptera: Curculionidae) reduces insecticide applications and increases cotton yield and economic return. Journal of Economic Entomology, v.98, p.1977-1983, 2005. DOI: $10.1093 /$ jee/98.6.1977.

SILVA, C.A.D.; RAMALHO, F.S. Kaolin spraying protects cotton plants against damages by boll weevil Anthonomus grandis Boheman (Coleoptera: Curculionidae). Journal of Pest Science, v.86, p.563-569, 2013. DOI: 10.1007/s10340-013-0483-0.

SISTERSON, M.S.; LIU, Y.B.; KERNS, D.L.; TABASHNIK, B.E. Effects of kaolin particle film on oviposition, larval mining, and infestation of cotton by pink bollworm (Lepidoptera: Gelechiidae). Journal of Economic Entomology, v.96, p.805-810, 2003. DOI: 10.1093/jee/96.3.805.

Recebido em 26 de janeiro de 2015 e aprovado em 15 de julho de 2015 